\title{
A novel multi-cell silicon drift detector for Low Energy X-Ray Fluorescence (LEXRF) spectroscopy
}

\author{
J. Bufon, ${ }^{a, b, c, 1}$ M. Ahangarianabhari, ${ }^{d, e}$ P. Bellutti, ${ }^{f}$ G. Bertuccio, ${ }^{d, e}$ S. Carrato, ${ }^{c}$ \\ G. Cautero, ${ }^{b, a}$ S. Fabiani, ${ }^{a}$ G. Giacomini, ${ }^{f}$ A. Gianoncelli, ${ }^{b, a}$ D. Giuressi, ${ }^{b, a}$ \\ M. Grassi, ${ }^{g, h}$ P. Malcovati, ${ }^{g, h}$ R.H. Menk, ${ }^{b, a}$ A. Picciotto, ${ }^{f}$ C. Piemonte, ${ }^{f}$ \\ I. Rashevskaya, ${ }^{a}$ A. Rachevski, ${ }^{a}$ A. Stolfa, ${ }^{b}$ A. Vacchi, ${ }^{a}{ }^{a}$ G. Zampa ${ }^{a}$ and N. Zampa ${ }^{a}$ \\ ${ }^{a}$ INFN Trieste, \\ Padriciano 99, I-34149, Trieste, Italy \\ ${ }^{b}$ Elettra-Sincrotrone Trieste S.C.p.A., \\ SS 14, I-34012, Trieste, Italy \\ ${ }^{c}$ University of Trieste, \\ Piazzale Europa 1, I-34128, Trieste, Italy \\ ${ }^{d}$ Politecnico di Milano, \\ Via Anzani 42, I-22100, Como, Italy \\ e INFN Milano, \\ Via Celoria 16, I-20133, Milano, Italy \\ ${ }^{f}$ Fondazione Bruno Kessler, \\ Via Sommarive 18, I-38123, Trento, Italy \\ ${ }^{g}$ University of Pavia, \\ Via Ferrata 1, I-27100, Pavia, Italy \\ ${ }^{h}$ INFN Pavia, \\ Via Agostino Bassi 6, I-27100, Pavia, Italy \\ E-mail: jernej.bufon@elettra.eu
}

RECEIVED: September 29, 2014

ACCEPTED: November 4, 2014

PUBLISHED: December 9, 2014

${ }^{1}$ Corresponding author. 


\section{Introduction}

It is very difficult to find in a synchrotron radiation facility two be amlines re quiring, in terms of detection of photons, electrons or other particles, exactly the same kind of device, with the same characteristics and performances. Even if limiting the analysis to X-ray Fluorescence Spectroscopy, it is possible to find fundamentally different requirements, in terms of photon energy range, geometry of the acquisition system, rate of events etc. This is the main reason why commercial detectors often fail to exploit the full potential that a synchrotron provides and this is why the development of customized detectors is needed.

For the above-mentioned reasons it was decided to proceed with the buildup of a new Silicon Drift Detector (SDD) devoted to the study of fluorescence e missions, which would be reconfigurable as much as possible, able to work in a very high range of energies, starting from very low energy $(200 \mathrm{eV})$, and able to front the major challenge presented by new generation sources, i.e. the dramatically high number of events to detect. The prototype we are presenting in this work is specialized to work at very low energies where the detection system presents major development difficulties, not only for reasons pertinent to the SDD sensitivity, but also since the system has to work in vacuum, which is necessary, at these energies, for every experimental activity. The vacuum involves many difficulties caused by heat dissipation and the transport of the signals out of the experimental chamber. Moreover at low energies the X-ray fluorescence yield is much lower that the one at high energies, causing a reduction of the emitted fluorescence signal.

In our project we have focused on the TwinMic beamline of Elettra Synchrotron which is particularly suited for bio-related research with low-Z elements [1]. The current commercial Low Energy X-Ray Fluorescence (LEXRF) detection setup consists of eight single-cell Silicon Drift Detectors (SDD) in an annular configuration [ 2]. The sensitive a rea that they cover results in a total of $230 \mathrm{~mm}^{2}$. The $8 \mathrm{SDD}$ are located at a distance of $28 \mathrm{~mm}$ from the specimen and tilted by $20^{\circ}$, so they cover together a solid angle which is about $4 \%$ of the hemisphere where the irradiated specimen emits fluorescence. This implies long and expensive scanning times (from 6 to 12 hours) and currently limits the performance of the beamline TwinMic, since large part of the fluorescence is lost. 

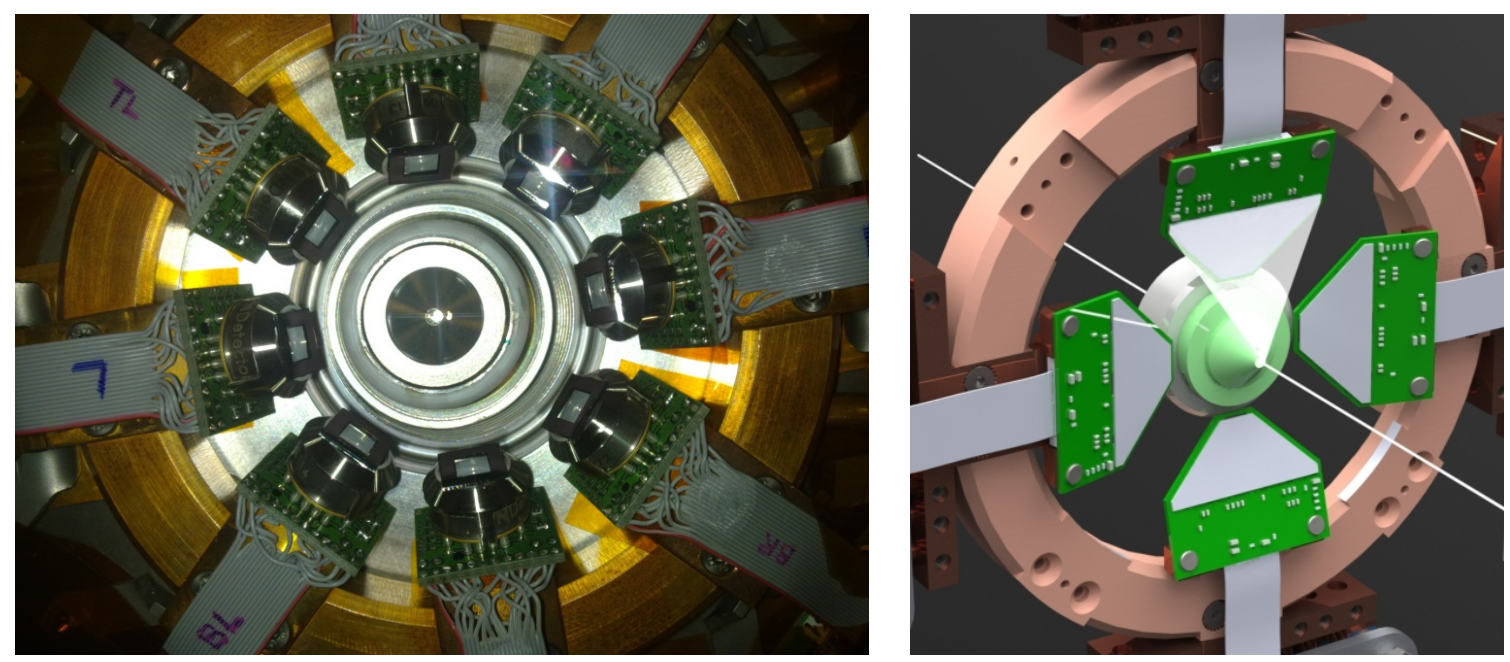

Figure 1. Picture of the current LEXRF detection system on the beamline TwinMic and a rendering of the new system consisting of 4 trapezoidal SDDs.

\section{The new detector}

The main goal of the new detector is therefore the increase of the solid angle coverage in order to drastically reduce the measurement time, thus saving on the cost of the experiments and reducing the degradation of the sample during the measurement. To achieve this it was decided to create a pixelated trapezoidal SDD. From extensive studies on the shape of the new sensor, it was found that the merge of 4 trapezoidal detectors gives a configuration that takes the best advantage of the space inside the TwinMic experimental chamber. The 4 detectors are configured as a side surface of a square pyramidal frustum: the photon beam passes through the top base while the specimen is placed over the bottom base (figure 1). The total active area of the 4 trapezoidal SDD is $912 \mathrm{~mm}^{2}$ with which the new system is able to cover up to $40 \%$ of the hemisphere in which the sample emits fluorescence. Thus we achieved an improvement of a factor of 10 in the solid angle coverage.

The new detector has additional advantages. Above all its partition into pixels; in fact small pixels allow to acquire events at higher frequency reducing the risk of pile-up, in addition of having a lower leakage current on the anode. Besides this, a pixelated detector allows to have, in addition to the information on the number and energy of the photons, also the spatial information about the point of incidence of the radiation. This could have interesting applications in the study of a specimen by providing information on the angle of the emitted fluorescence. Although the fluorescence is, in fact, isotropic, the sample may radiate in different ways in the various directions if its surface is not perfectly planar. The solid angle of the radiation, obtained in the measurements, would thus provide morphological information of the specimen surface.

The trapezoidal detectors, jointly designed by INFN Trieste and FBK, were divided into 28 pixels, 20 squared and 8 triangular, with an area of $9.5 \mathrm{~mm}^{2}$ and $4.75 \mathrm{~mm}^{2}$ respectively. The number of pixels is not, however, a set parameter. We are also studying trapezoids with fewer cells and with hexagonal pixels instead of squared ones, aiming at the highest configurability and adaptability of the detector. 


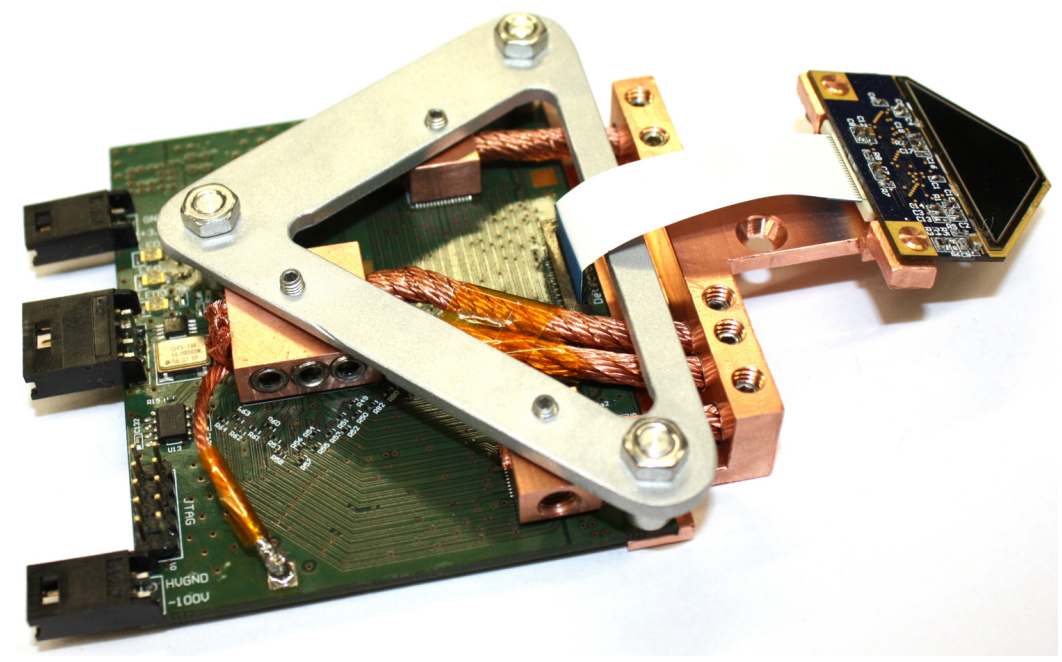

Figure 2. Picture of the first prototype; on the right the PCB with the SDD and the two ASICs and on the left the back-end PCB with the two ADCs and the FPGA.

\section{The first prototype}

To check the quality of the idea and the feasibility of the project a prototype (figure 2) was built. The first system consists of a single trapezoidal SDD and its read-out e lectronics. To have a quick response, especially regarding the critical points (i.e. the mechanical assembly and the electronics operating in vacuum), it was decided to use, for the production of the detector carried out by FBK, part of the periphery of the wafers dedicated to the LOFT space project [3]. The silicon run was obviously not optimized for LEXRF applications and indeed had a low efficiency in the detection of low energy radiation.

The read-out electronics relied on two 32-channel ASICs, designed by the Politecnico di Milano, also dedicated to LOFT. Each channel contains a charge sensitive amplifier (CSA), a configurable a nalog s haper, a peak s tretcher, a mplitude $d$ iscriminator and c ontrol logic for pile-up rejection and channel reset, finally a multiplexer provides a single output for all the 32 channels of the ASIC [4]. We used two ASICs, even though one would be sufficient for the 28 pixels of a trapezoidal SDD, in order to minimize as much as possible the length of the connections between the detector anodes and the CSAs inside the ASIC, and consequently reduce the parasitic capacitance introduced by the bonding wires. The output signal of the two ASICs is transmitted to a back-end PCB where it is sampled by two ADCs managed by an FPGA that processes the data and sends them to a PC outside the experimental chamber.

Such an assembled prototype has allowed us to quickly verify the goodness of the approach by mounting this first acquisition setup on the beamline, checking the mechanical dimensions and testing the electronics operating in vacuum $\left(10^{-6} \mathrm{mbar}\right)$.

\section{Preliminary tests}

Initial testing of the first prototype were made by irradiating the detector with a ${ }^{55} \mathrm{Fe}$ radioactive source in a climatic chamber, bringing the system down to $-20^{\circ} \mathrm{C}$. The main purpose of these 


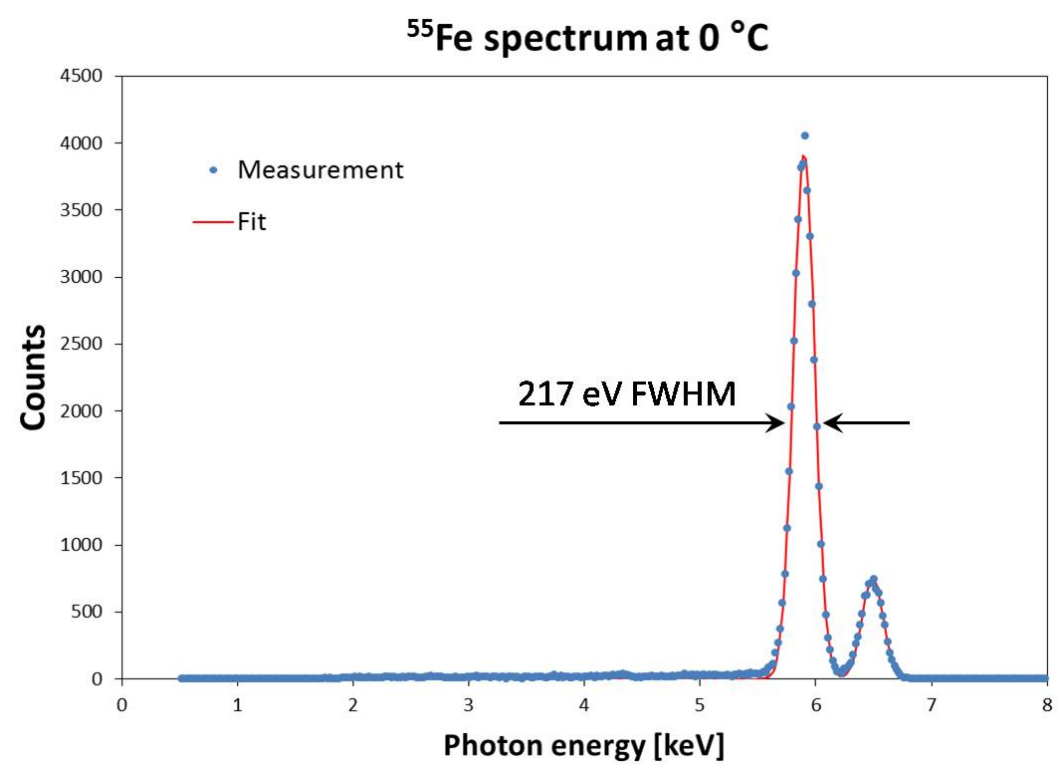

Figure 3. Spectrum of the ${ }^{55} \mathrm{Fe}$ radioactive isotope. In the fit, acquired at $0^{\circ} \mathrm{C}$, are clearly seen the two peaks $\mathrm{K} \alpha(5.9 \mathrm{keV})$ and $\mathrm{K} \beta(6.5 \mathrm{keV})$.

preliminary tests was to check both the count-rate and the resolution of the new system. Also in this case it was only an indicative estimation since the energy resolution was limited by the ASIC, designed for the detection of much higher energies with a severe power consumption constraint for the huge LOFT detection system.

After the characterization in the climatic chamber, we proceeded with the installation of the prototype on the TwinMic beamline. The mechanical installation succeeded smoothly, confirming the measurements made at the beginning of the project. At this point the main challenge was the in-vacuum cooling of the system, as the back-end electronics, which consists of an FPGA and two ADCs, dissipates almost $5 \mathrm{~W}$. The problem has been solved by exploiting the cooling ring already present in the experimental chamber. The chiller, which circulates a mixture of water and alcohol, was able to bring the system down to $-10^{\circ} \mathrm{C}$. After the first functionally tests without the beam, a known specimen was positioned in the experimental chamber. By moving with the beam across the sample we were able to detect the $\mathrm{Si}(1740 \mathrm{eV}), \mathrm{Al}(1486 \mathrm{eV}), \mathrm{Mg}(1254 \mathrm{eV})$ and a small peak of $\mathrm{F}(677 \mathrm{eV})$.

The first tests $\mathrm{g}$ ave the expected r esults, y ielding a resolution of $217 \mathrm{e} \mathrm{V}$ at the ${ }^{55} \mathrm{Fe}\left(0^{\circ} \mathrm{C}\right)$, shown in figure 3 , and glimpse energies down to the $\mathrm{F}$ emission line. We also confirmed the proper operation of the electronics and the cooling of the whole system in the vacuum.

\section{Conclusions and outlook}

The first prototype tested on the TwinMic beamline of Elettra was very helpful to front and solve the problems related to the new detection system placed under vacuum. The trapezoidal configuration covers about 10 times more solid angle with respect to the present commercial setup and preliminary measurements showed that the major limits in terms of energy resolution, as foreseen, 
are constituted by the SDD and ASIC systems that were developed for completely different applications.

On the basis of these tests an improved version of the system is already being planned. The new setup will consist of a new trapezoidal SDD from a dedicated run with a thin entrance window, so providing very high efficiency at low energies, and an extremely low-noise single-channel ASIC designed by Politecnico di Milano, which will be placed very close to the anode in order to minimize the length of the wire bonds. The first tests of the new system will be carried out as early as in December 2014 during a dedicated beamtime on the TwinMic beamline.

\section{Acknowledgments}

The authors thankfully acknowledge the work of the Elettra TwinMic beamline team for their help in the preparation of the experimental setups and tests. Finally, this work would not have been possible without the financial support of the authors' funding institutions, INFN through the CSN5 ReDSoX project and Elettra Sincrotrone Trieste.

\section{References}

[1] B. Kaulich et al., Low-energy X-ray fluorescence microscopy opening new opportunities for biorelated research, J. R. Soc. Interface 6 (2009) S641.

[2] A. Gianoncelli, G. Kourousias, A. Stolfa and B. Kaulich, Recent developments at the TwinMic beamline at ELETTRA: an 8 SDD detector setup for low energy X-ray fluorescence, J. Phys. Conf. Ser. 425 (2013) 182001.

[3] M. Feroci et al., The Large Observatory for X-ray Timing (LOFT), Exp. Astron. 34 (2012) 415.

[4] M. Ahangarianabhari et al., A low-power CMOS ASIC for X-ray silicon drift detectors low-noise pulse processing, 2014 JINST 9 C03036. 